\title{
Urticária pigmentosa no adulto: tratamento bem-sucedido em associação com fototerapia*
}

\section{Adult urticaria pigmentosa: successful treatment in association with phototherapy}

\author{
Rafael Capelo Costa ${ }^{1}$, Juliana D'Andrea Molina ${ }^{2}$, Marilda Aparecida Milanez Morgado de Abreu ${ }^{3}$
}

Costa RC, Molina JD, Morgado de Abreu MAM. Urticária pigmentosa no adulto: tratamento bem-sucedido em associação com fototerapia / Adult urticaria pigmentosa: successful treatment in association with phototherapy. Rev Med (São Paulo). 2020 jul.-ago.;99(4):400-4.

RESUMO: Introdução: A urticária pigmentosa em adultos é uma forma de mastocitose rara e persistente, frequentemente com manifestações sistêmicas e acometimento da medula óssea, e manifestações cutâneas menos evidentes, ao contrário do que ocorre na infância. Objetivo: Relatar um caso de início na fase adulta, com lesões cutâneas leves, que evoluiu com sintomas de liberação histamínica, porém sem acometimento sistêmico. Metodologia: As informações foram obtidas através de revisão do prontuário, entrevista com o doente, registro fotográfico e revisão da literatura. Conclusão: O diagnóstico correto e a investigação precoce do acometimento de outros órgãos são importantes, bem como o tratamento adequado e prevenção de reações anafilactoides. A fototerapia é opção interessante de tratamento nos casos refratários.

Descritores: Urticária pigmentosa; Mastocitose cutânea; Mastocitose sistêmica.

\begin{abstract}
Introduction: Adult urticaria pigmentosa is a rare and persistent form of mastocytosis, often with systemic manifestations and involvement of the bone marrow, and less evident cutaneous manifestations, contrary to what occurs in childhood. Objective: The authors aim to report a case of adult onset, with mild skin lesions, and development of symptoms of histamine release, without systemic involvement. Methodology: The information was obtained through review of the medical record, interview with the patient, photographic record, and a literature review. Conclusion: The correct diagnosis and the early assessment of involvement of other organs is important, as well as adequate treatment and prevention of anaphylactoid reactions. Phototherapy is an interesting treatment option for refractory cases.
\end{abstract}

Keywords: Urticaria pigmentosa; Mastocytosis, cutaneous; Mastocytosis, systemic.

\footnotetext{
* Trabalho realizado no Hospital Regional de Presidente Prudente, SP, Universidade do Oeste Paulista.

1.Médico dermatologista pela SBD, Clínica privada. ORCID: https://orcid.org/0000-0002-3224-3336. E-mail: capelo@gmail.com.

2. Médica dermatologista pela SBD, Clínica privada. ORCID: https://orcid.org/0000-0001-9261-5739. E-mail: julianadandrea@gmail.com.

3.Médica dermatologista pela SBD, mestrado e doutorado em ciências. Serviço de Dermatologia do Hospital Regional de Presidente

Prudente/Universidade do Oeste Paulista. ORCID: https://orcid.org/0000-0001-9099-6013. E-mail: marilda@morgadoeabreu.com.br.

Endereço para correspondência: Rafael Capelo Costa, Avenida Santos Dumont, 2727 - sala 301. Fortaleza, CE. CEP: 60150-161.
} 


\section{INTRODUÇÃO}

Vastocitose é doença rara, com prevalência de 2 casos por 300.000 pacientes em consulta dermatológica, e caracteriza-se pela proliferação e acúmulo de mastócitos na pele e/ou outros órgãos e tecidos, principalmente medula óssea e trato gastrintestinal ${ }^{1}$. Subdivide-se em mastocitose cutânea (MC), mais frequente na infância, e mastocitose sistêmica (MS), de início mais tardio, a partir da terceira década. Na última, há acúmulo de mastócitos em outros órgãos e tecidos, podendo ser progressiva e fatal, se associada à malignidade ${ }^{2}$. A expressão da forma cutânea é rara em adultos, tornando escassos os relatos sobre o seu curso em pacientes de meia-idade.

As manifestações clínicas relacionam-se à liberação de mediadores pró-inflamatórios por mastócitos ativados e pela infiltração de diferentes órgãos pelos mastócitos ${ }^{1}$.

O prognóstico depende de vários fatores, mas nos casos do adulto, as lesões cutâneas persistem, regredindo apenas na minoria dos casos. A maioria apresenta curso estável e expectativa de vida normal, sendo a evolução para formas agressivas excepcional ${ }^{3}$.

Apresentamos um caso raro de mastocitose em adulto, com lesões e sintomas restritos à pele, e sem acometimento sistêmico confirmado por intensa investigação clínica e laboratorial.

\section{RELATO DE CASO}

Doente masculino, 36 anos, branco, queixava-se de lesões na pele há um ano, inicialmente nos antebraços, progredindo, em quatro meses, para o tronco.

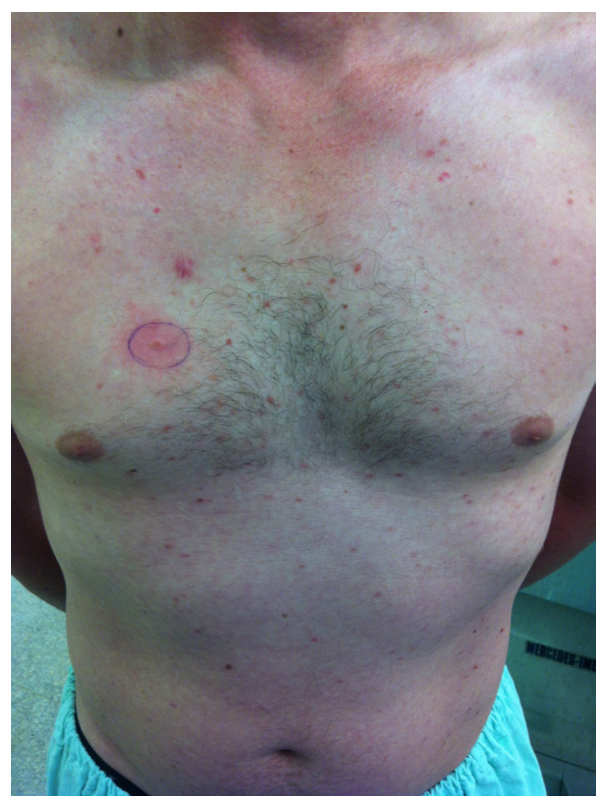

Figura 1: Pápulas eritematosas com sinal de Darier positivo.
Ao exame evidenciavam-se pequenas pápulas eritêmato-edematosas, que desapareciam à digitopressão, na face anterior dos antebraços e no tronco, com presença do sinal de Darier (Figuras 1 e 2). Lesões mais antigas eram acastanhadas e não regrediam à digitopressão (Figura 3). Havia prurido e sensação de queimação. Em consultas posteriores, referiu cólicas abdominais, tontura e taquicardia.

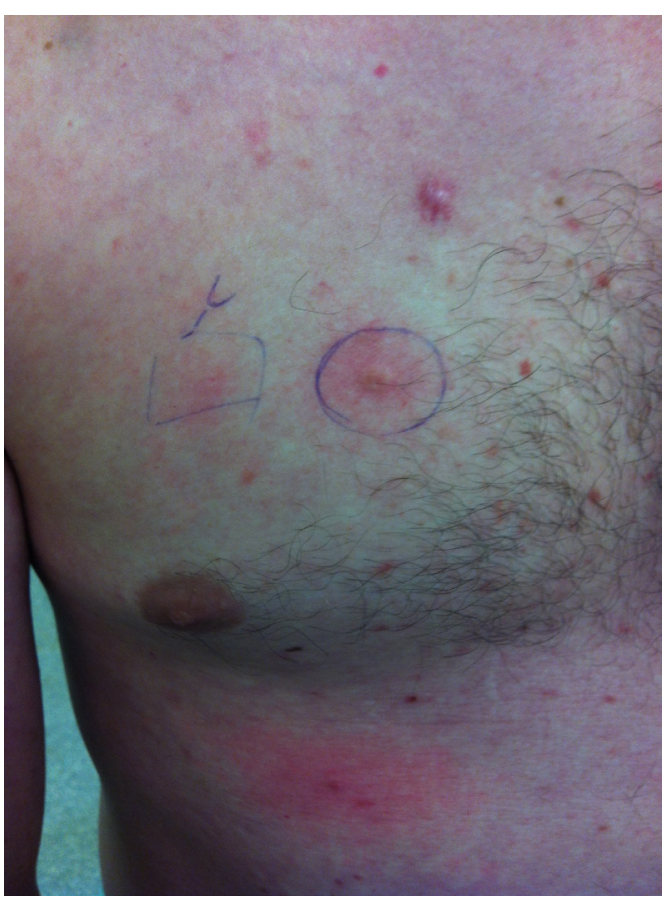

Figura 2: Detalhe do sinal de Darier.

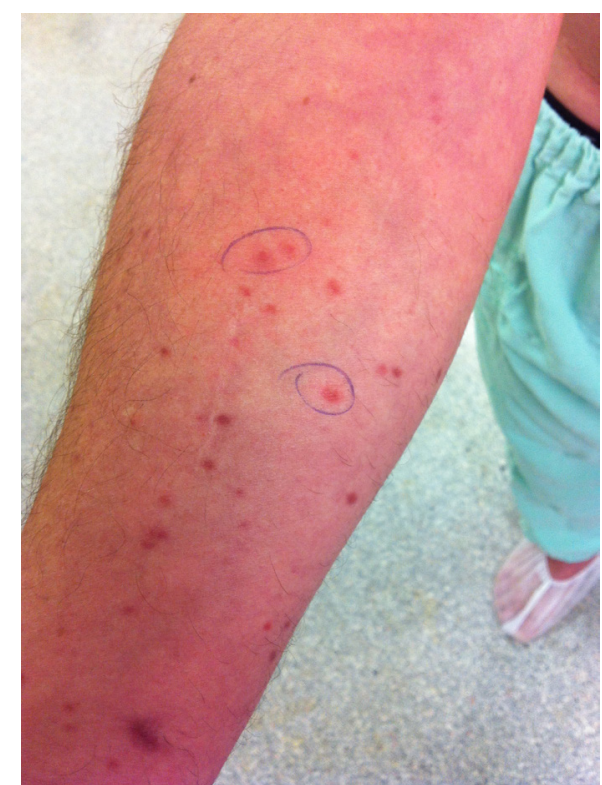

Figura 3: Pápulas acastanhadas mais antigas

Negava doenças prévias e história de doença dermatológica familiar. 
Avaliação laboratorial sem alterações, inclusive triptase sérica (Tabela 1). A histopatologia de lesão evidenciou dermatite perivascular superficial residual, com presença de 9 mastócitos em campo de grande aumento (Figura 4). Foi encaminhado para cardiologia, gastroenterologia e hematologia, e foi ealizada investigação com exames de imagem, aspirado de medula óssea e eletrocardiograma, sem nenhuma alteração.

Tabela 1: Exames mais relevantes

\begin{tabular}{l|l}
\hline Exame & Resultado \\
\hline LDH & 522 U/L( VR =313-618) \\
Triptase & $\begin{array}{l}13,1 \mathrm{ug} / \mathrm{L} \text { (inferior a 13,5) } \\
\text { Raros mastócitos anômalos } \\
\text { Mielograma }\end{array}$ \\
Imunofenotipagem de sangue periférico & $\begin{array}{l}\text { Sem incremento de mastócitos na medula } \\
\text { Biópsia de medula óssea }\end{array}$ \\
Radiografias: coluna lombo-sacra, torácica, cervical, bacia, crânio, tórax & $\begin{array}{l}\text { Sem alterações significativas } \\
\text { Ecografia de abdome }\end{array}$ \\
\hline
\end{tabular}

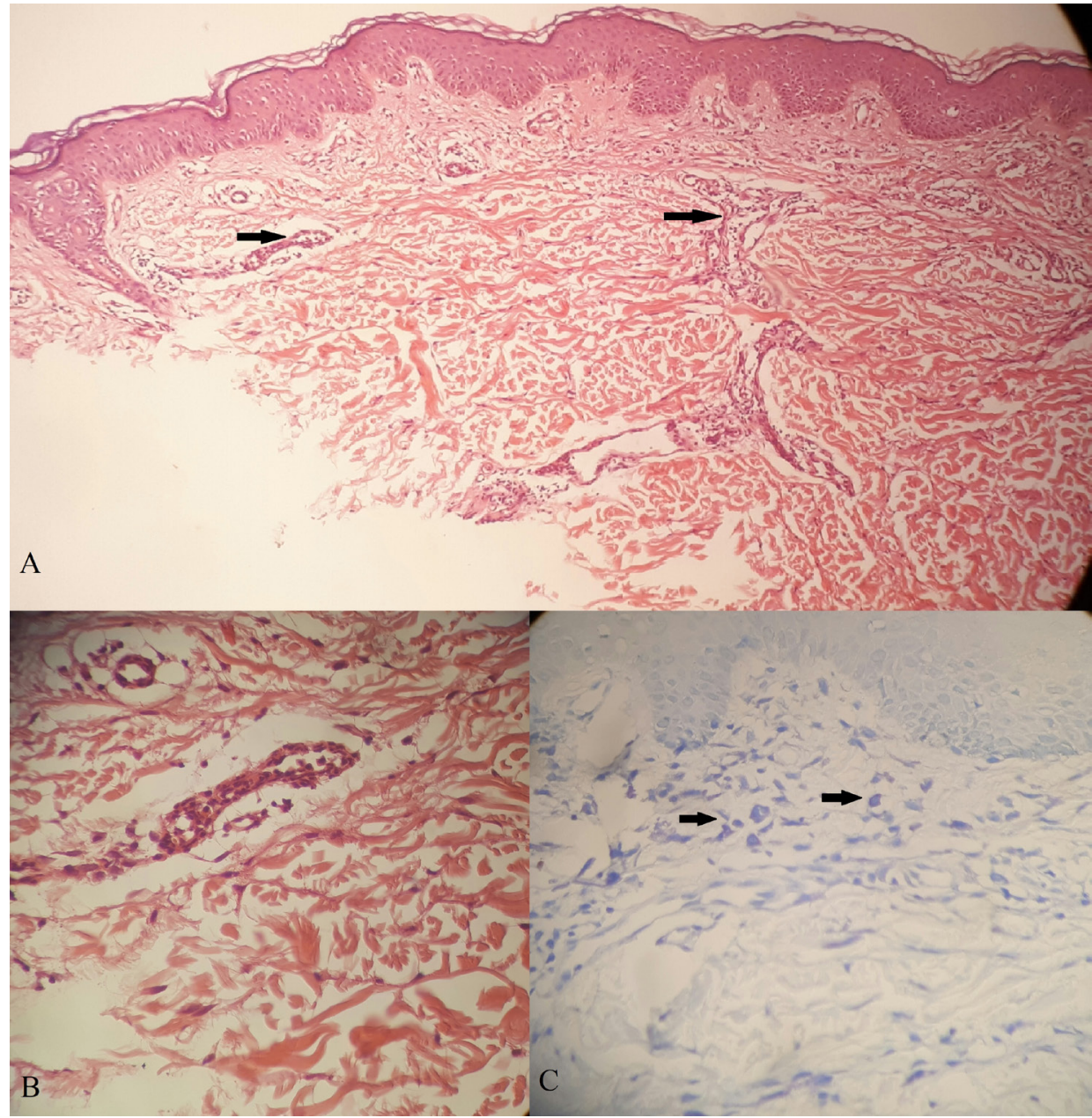

Figura 4: Fotomicroscopia da pele. A - Derme com infiltrado inflamatório perivascular (setas) (Hematoxilina-Eosina, aumento de 100x). B - Detalhe do infiltrado linfocitário perivascular (Hematoxilina-Eosina, aumento de 400x). C - Presença de 9 mastócitos na derme superficial (setas) (Azul de Toluidina, aumento de 400x)

Foi iniciado tratamento com anti-histamínicos, estabilizador de mastócitos e anti-leucotrienos. Em sua evolução, apresentou dificuldade no controle dos sintomas e lesões cutâneas, apesar da combinação medicamentosa (Tabela 2). 
Costa RC, et al. Urticária pigmentosa no adulto: tratamento bem-sucedido em associação com fototerapia.

Tabela 2: Terapêutica sistêmica utilizada, na ordem cronológica das drogas, e respectivas dose, frequência e tempo de uso

\begin{tabular}{l|l|l|l}
\hline Medicação (ordem cronológica) & Dose & Frequência & Tempo de uso \\
\hline Loratadina & $10 \mathrm{mg}$ & $2 \mathrm{x} / \mathrm{d}$ & 6 meses \\
Cetotifeno +loratadina & $1 \mathrm{mg}+10 \mathrm{mg}$ & $2 \mathrm{x} / \mathrm{d}$ & 8 meses \\
Montelucaste + cetotifeno + ebastina & $10 \mathrm{mg}+1 \mathrm{mg}+10 \mathrm{mg}$ & $1 \mathrm{x} / \mathrm{d}+2 \mathrm{x} / \mathrm{d}+2 \mathrm{x} / \mathrm{d}$ & 5 meses \\
Dexclorfeniramina + fexofenadina* & $2 \mathrm{mg}+180 \mathrm{mg}$ & $3 \mathrm{x} / \mathrm{d}+1 \mathrm{x} / \mathrm{d}$ & 8 meses $^{*}$ \\
\hline
\end{tabular}

*-Associado à fototerapia após esse período.

Optou-se por associar fototerapia com UVB-NB (ultravioleta tipo B de banda estreita - narrow band - faixa compreendida entre 311-312nm), e a partir da sexta sessão já foi alcançado resultado satisfatório, com desaparecimento das pápulas eritêmato-edematosas e do prurido (Figura 5). Foi utilizada cabine mista Prolumina - 21 lâmpadas UVBNB. Dose inicial de $50 \mathrm{~mJ} / \mathrm{cm}^{2}$ (33"), com aumento de 50 $\mathrm{mJ}$ por sessão, de acordo com efeitos adversos, às terças e sextas. Atingiu dose máxima de $1050 \mathrm{~mJ}$ (11'40"). Os sintomas sistêmicos remitiram conforme houve melhora das lesões cutâneas. Recebeu alta após 55 sessões, dose acumulada de $33 \mathrm{~J} / \mathrm{cm}^{2}$. Foi mantida dexclorfeniramina e fexofenadina durante as sessões e após alta. Após um mês apresentou recidiva do quadro, precisando reiniciar o tratamento.

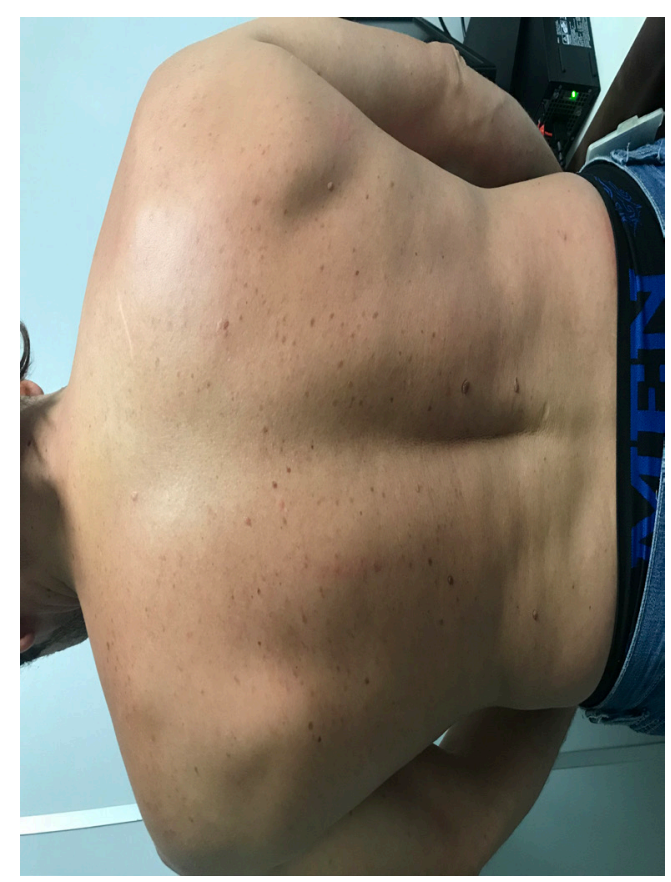

Figura 5: Boa resposta com associação da fototerapia UVB-NB

\section{DISCUSSÃO}

A mastocitose cutânea é definida pela erupção típica, critério maior, e um ou dois dos critérios menores: infiltrados monomórficos com grandes agregados triptase-positivos, ou mais de 20 mastócitos por campo microscópico; ou pela detecção da mutação KIT no códon 816 na pele lesada. Após, seguir um algoritmo para diferenciar MC da MS, abrangendo desde formas indolentes à leucêmicas ${ }^{4}$.

Pacientes pediátricos, geralmente, são acometidos por MC e adultos por MS, sendo indicada a realização de exame da medula óssea nos últimos, mesmo com triptase sérica normal ${ }^{4}$.

A MC subdivide-se em urticária pigmentosa, mastocitose cutânea difusa e mastocitoma solitário na pele, e ainda, o subtipo telangiectasia macular eruptiva perstans. A urticária pigmentosa é a forma mais comum, caracterizada por máculas ou pápulas vermelho-acastanhadas, de 3-4 $\mathrm{mm}$, simétricas no tronco e nas coxas. O sinal de Darier (formação de urtica após fricção da lesão) está presente ${ }^{2}$.

Manifestações paroxísticas são devidas à degranulação de mastócitos: ruborização corporal, eritema pruriginoso, cefaleia, palpitações, hipotensão, síncope, dispneia, precordialgia, diarreia, parestesias, prurido, urticária, broncoespasmo e bolhas ${ }^{1}$.

Sugerem MS: hemograma com anemia, leucocitose e eosinofilia, aumento nos níveis de histamina urinária e triptase sérica $>20 \mathrm{ng} / \mathrm{mL}$, hepatoesplenomegalia ou linfonodomegalia, osteoporose ou infiltrados de mastócitos na medula ${ }^{1}$.

O tratamento visa aliviar os sintomas tais como prurido (emprego de antagonistas H1), e manifestações gastrintestinais (antagonistas H2) e evitar fatores desencadeantes de degranulação dos mastócitos a saber: fricção, mudança brusca da temperatura, exercício físico intenso, estresse, anestesia geral, bebida alcoólica, anti-inflamatório não esteroidal, narcóticos, contraste radiológico e radiação ionizante. É importante portar epinefrina, corticoides e anti-histamínicos pelo risco de reação anafilactoide. Nesse, contexto, medicamentos como cromoglicato de sódio e cetotifeno estabilizam a membrana dos mastócitos. Para os mastocitomas, corticóides oclusivos ou excisão. Na MS, podem ser usadas drogas citorredutoras. Atualmente, os imunobiológicos omalizumabe e imatinibe vêm sendo estudados em casos graves com anafilaxia e doença sistêmica ${ }^{4,5}$.

A fototerapia já é utilizada na dermatologia desde a virada do século XIX, inicialmente para tratamento de psoríase. Hoje é utilizada em diversas patologias cutâneas, especialmente as que estão associadas ao prurido. A UVB 
tem penetração na epiderme, com $10 \%$ da dose atuando na junção dermo-epidérmica. A PUVA penetra até a derme, com $30 \%$ da dose atuando nessa camada 6 .

A fototerapia é tratamento de segunda linha para lesões cutâneas resistentes, seja na MC ou MS indolente. As alterações induzidas por UV nos mastócitos cutâneos são pouco conhecidas. A luz UV suprime a liberação de histamina pelos mastócitos ativados, além de induzir apoptose. Tanto PUVA quanto UVB-NB apresentam resultados satisfatórios em estudos. Brazelli et al. ${ }^{7}$ obtiveram remissão completa do prurido em $35 \%$ dos doentes e melhora substancial das manifestações cutâneas em todos eles, tratados com PUVA ou UVB-NB. Resultados mais rápidos e com melhor controle das manifestações cutâneas e sintomas foram alcançados com PUVA. Já com UVB-NB obtiveram bons resultados com menor exposição UV e melhor tolerabilidade ${ }^{7}$.
Devido ao aparecimento tardio do quadro perante o contexto epidemiológico da urticária pigmentosa, foi realizada exaustiva investigação do acometimento de outros órgãos. Os sintomas sistêmicos apresentados pelo doente foram relacionados à liberação de histamina, já que regrediram com o controle das lesões de pele.

\section{CONCLUSÃO}

O relato destaca a importância de diferenciar os casos de MC e MS objetivando afastar quadros agressivos, orientar o seguimento e proporcionar melhora dos sintomas. A associação da fototerapia ao tratamento, seja UVBNB ou PUVA, deve ser considerada em casos refratários ao tratamento convencional, pois poderá trazer grande benefício ao doente.

Participação dos autores: Costa $R C$ : Revisão de literatura, redação do texto, correções iniciais, formatação e submissão à revista. Molina JD: Revisão de literatura, redação e atualização do texto, submissão no comitê de ética e pesquisa. Morgado de Abreu MAM: Orientadora, correções e revisão final.

Conflito de interesse: Nenhum conflito

\section{REFERÊNCIAS}

1. Barete S. Les mastocytoses. Ann Dermatol Venereal. 2014;141:698-714. doi: https://doi.org/10.1016/j. annder.2014.08.002.

2. Barros JA, Maluf LC, Machado Filho CD. Mastocitose. An Bras Dermatol. 2009;84(3):213-25. doi: https://doi. org/10.1590/S0365-05962009000300002.

3. Brockow K. Epidemiology, prognosis, and risk factors in mastocytosis. Immunol Allergy Clin North Am. 2014;34(2):283-95. doi: https://doi.org/10.1016/j. iac.2014.01.003.

4. Valent P, Akin C, Escribano L, Födinger M, Hartmann $\mathrm{K}$, Brockow K, et al. Standards and standardization in mastocytosis: consensus statements on diagnostics, treatment recommendations and response criteria. Eur J Clin Invest. 2007;37:435-53. doi: https://doi.org/10.1111/j.13652362.2007.01807.x.
5. Czarny J, Lange M, Lugowska-Umer H, Nowicki RJ. Cutaneous mastocytosis treatment: strategies, limitations and perspectives. Adv Dermatol Alergol. 2018;35(6):541-5. doi: https://doi.org/10.5114/ada.2018.77605.

6. Schneider LA, Hinrichs R, Scharffetter-Kochanek K. Phothotherapy and photochemotherapy. Clin Dermatol. 2008;26:464-76. doi: https://doi.org/10.1016/j. clindermatol.2007.11.004.

7. Brazzelli V, Grassi S, Merante S, Grasso V, Ciccocioppo R, Bossi G, et al. Narrow-band UVB phototherapy and psoralen-ultraviolet A photochemotherapy in the treatment of cutaneous mastocytosis: a study in 20 patients. Photodermatol Photoimmunol Photomed. 2016;32:238-46. doi: https://doi. org/10.1111/phpp.12248.

Submetido: 23.02.2018

Aceito: 29.06.2020 